\title{
Armada: A Flagship Multi-Mission Architecture
}

A Planetary Science White Paper

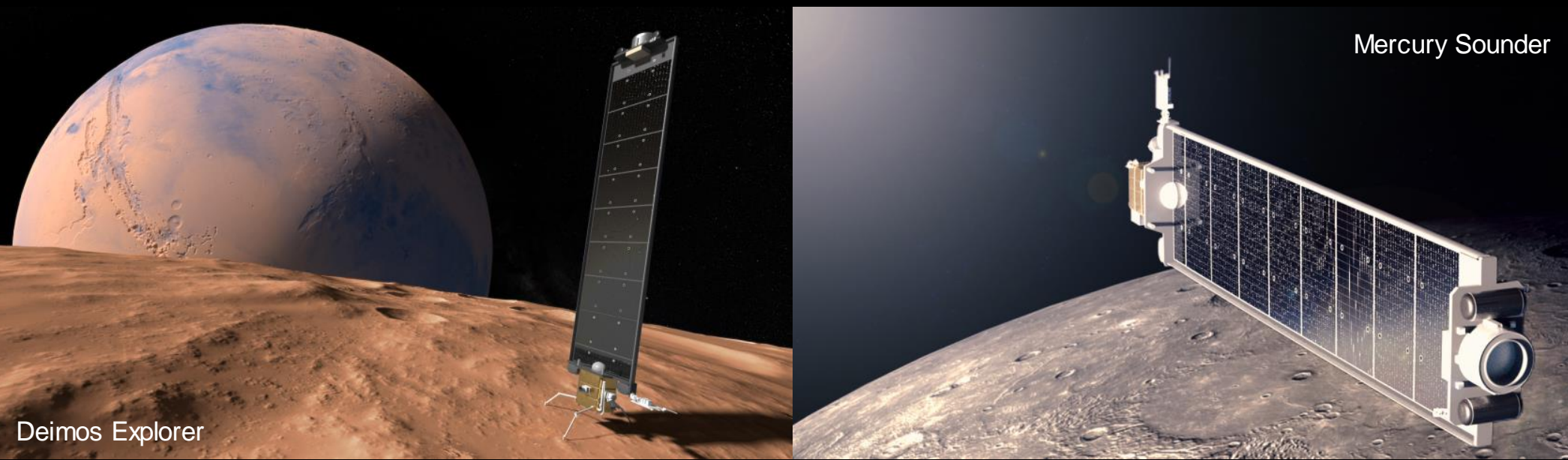

David Paige (University of California, Los Angeles) dap@moon.ucla.edu

Ceres Water Mapper

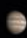
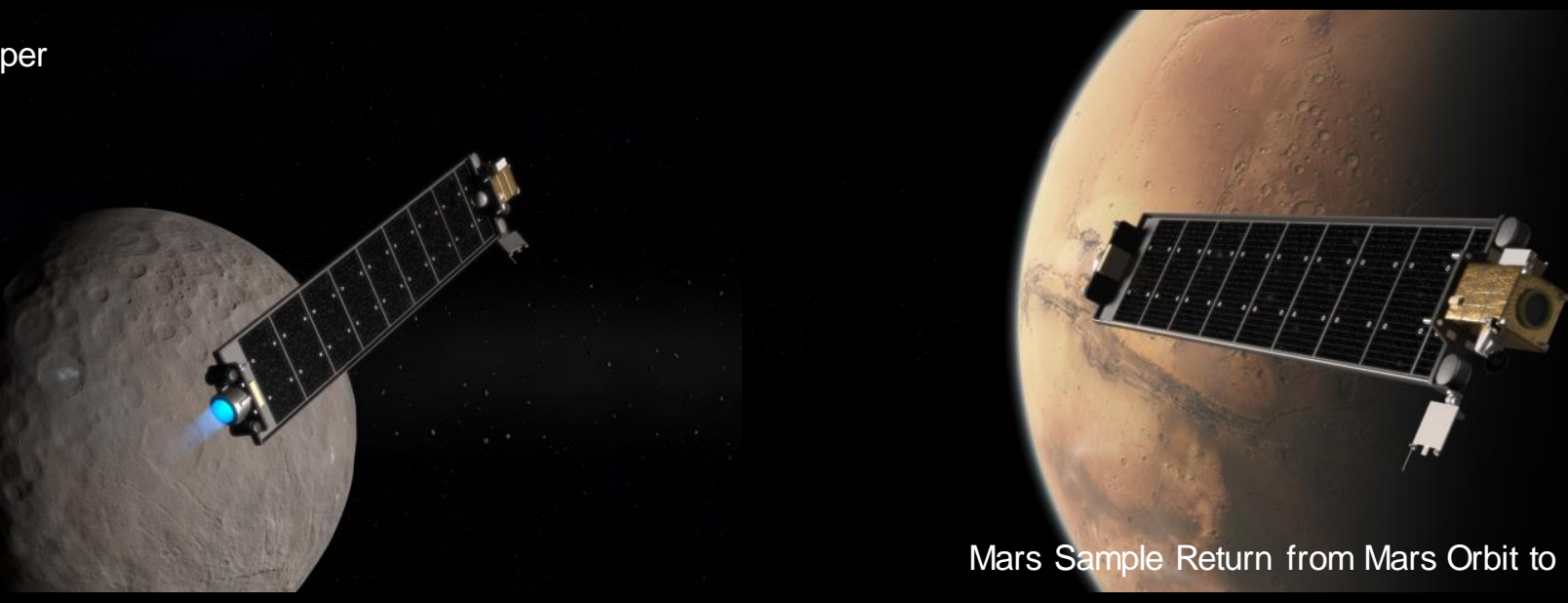

Mars Sample Return from Mars Orbit to Earth Orbit

Stephen Bailey (Deep Space Systems, Littleton, CO) steve.bailey@deepspacesystems.com 
Imagine a Flagship program that echoes the golden age of exploration, in which a fleet of vehicles are dispatched in all directions to the distant regions of human knowledge and experience. This white paper explores the idea of a NASA Planetary Science Flagship Program that, rather than focusing on a single destination, intentionally includes and involves the entire planetary science community in launching such an Armada, a fleet of science exploration vehicles dispatched to many deep space locations.

It is amazing to see the creativity and genuinely inspiring work done by the Planetary Science community as evidenced in the responses to this Decadal Survey and in papers, posters, and proposals by the hundreds. There are so many worthy ideas and scientists at all stages of their careers, with so much to contribute. We should all, as a community, be trying to figure out how to do more, how to involve more, how to include more. More people, more instruments, more destinations, more opportunities.

Throughout this paper, mission concepts too ambitious for CubeSats are depicted as examples of what might collectively be accomplished by the approach we advocate. Although we cannot (yet) perform this class of mission with a CubeSat, these are all within the range of a $200 \mathrm{~kg}$ wet mass system launched as a series of secondary payloads (rideshare) on any launch vehicle bound to Geo Transfer Orbit.

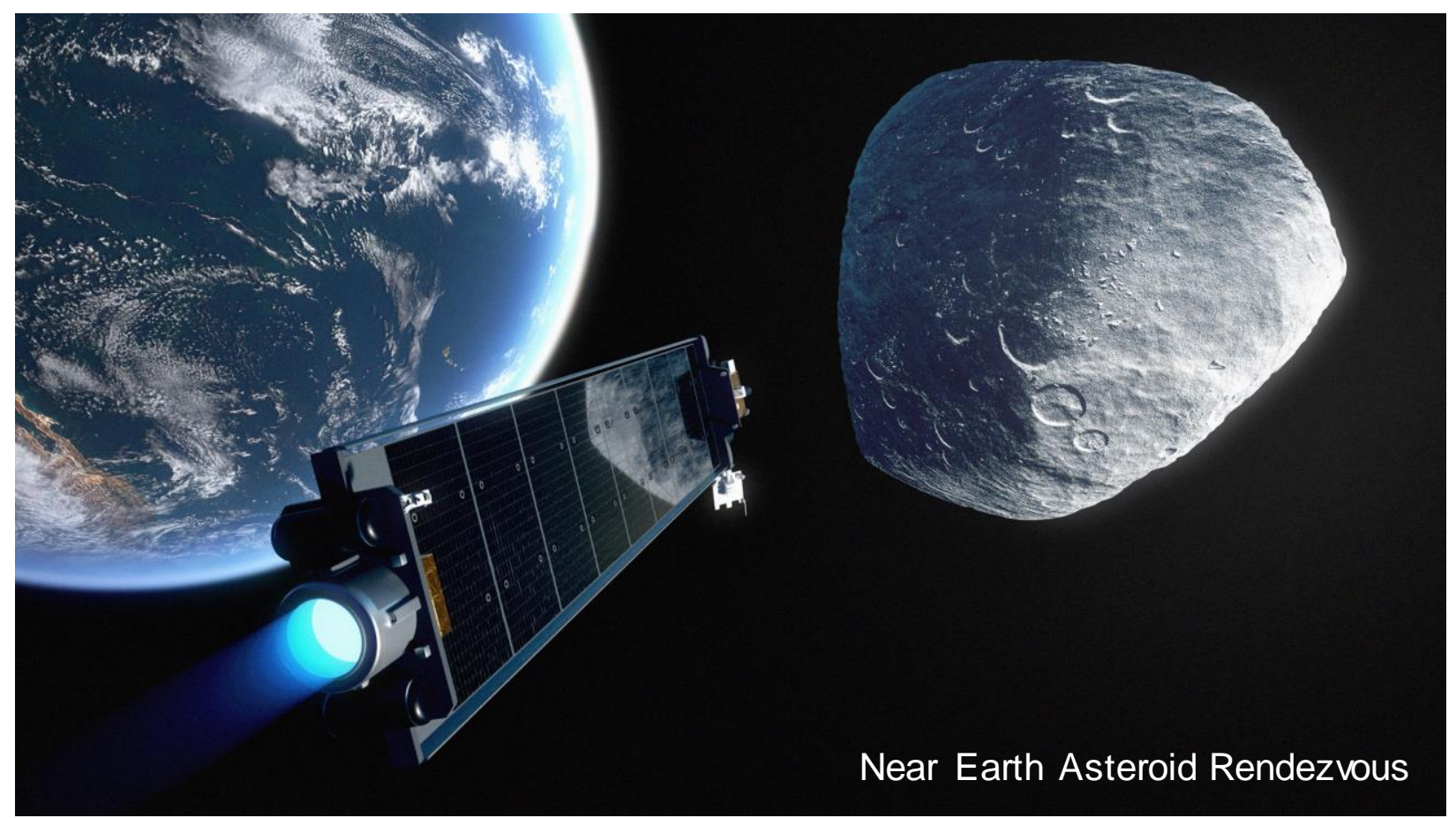

We are proposing Armada, a fleet of 20 small spacecraft with a high-performance gridded ion propulsion system and a propellant mass fraction sufficient to reach outer planet destinations. Within the Armada program, 20 Principal Investigators are selected to lead 20 teams comprised of some mix of government, academia, industry, and international teammates. Each PI is selected based on the strength of their science mission and the credibility of their team to accomplish what they propose.

The benefits to science of the Armada mission concept are as significant as they are numerous. At the most general level, one of the key strengths of the field of planetary science is the potential to understand solar system objects not simply as individuals, but to study multiple examples of these objects and make comparisons. The physical and chemical principals that governed the formation and 
evolution of these diverse bodies are exactly the same, but the outcomes can be radically different. The understanding we achieve by studying multiple bodies significantly enhances our understanding of the general problem of how planets, moons, comets and asteroids form, as well as their potential to support the origin and/or evolution of life. It also greatly helps us build scientific bridges to the community of astronomers and planetary scientists studying exoplanets. The notion that there is great scientific potential to explore new objects should be self evident. However, it has been said by some that "the easy planetary missions have all been done already". This may be true to some extent, but just because we've taken some images and gathered some limited data does not mean that we truly understand these bodies or their histories. For return trips, armed with the knowledge gained by earlier encounters, we can much better choose the best investigations and instrumentation with which to employ for the second, and third wave of exploration, now that we know what we're looking for. Additionally, there is also the possibilities of ties between Armada missions, and multi-decadal programs within NASA such as sample return, as well as international collaboration.

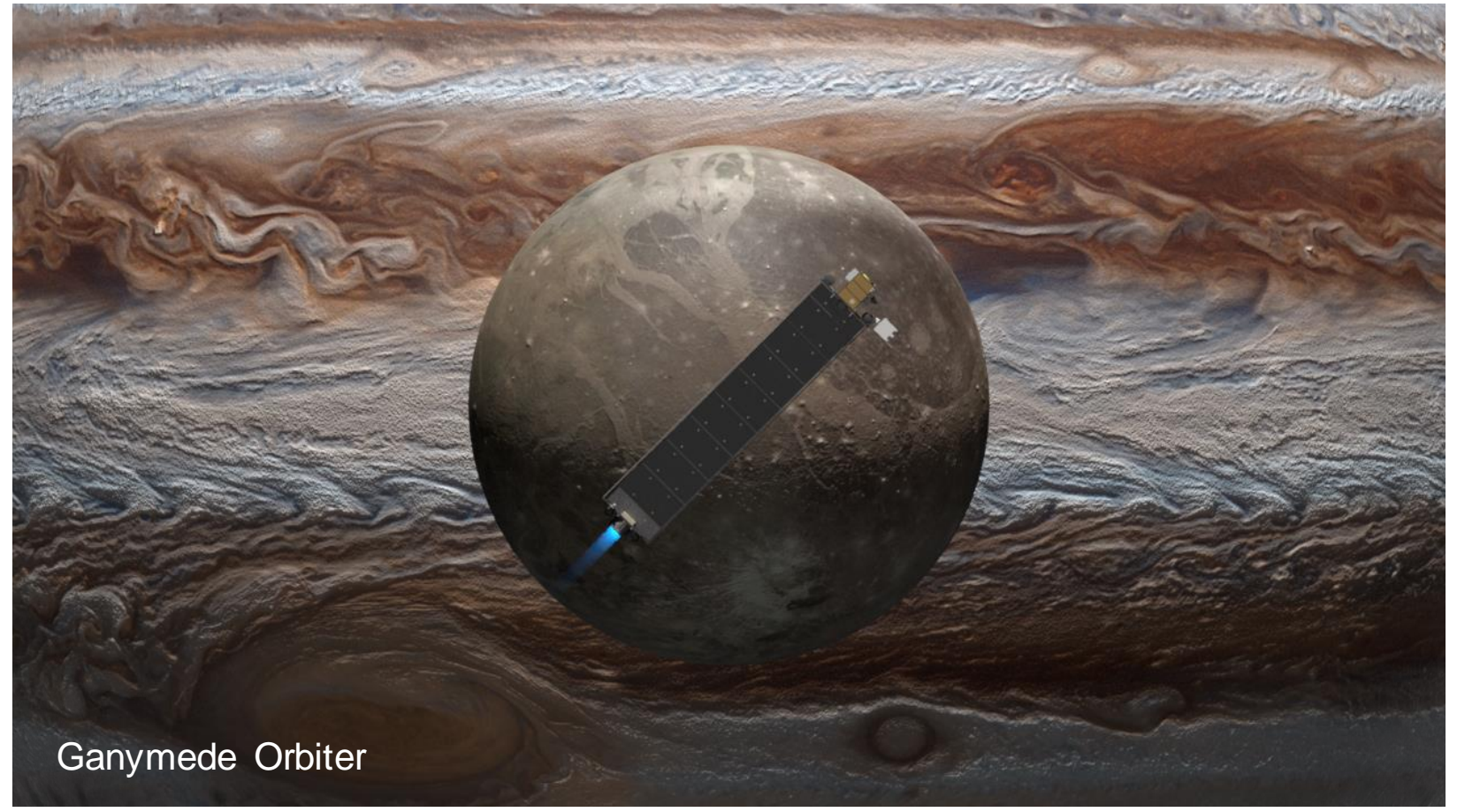

The benefits to the planetary science community of the Armada concept are also significant. Every decade, the science community is forced to decide between a limited number of choices for Flagship and New Frontiers-class missions. In the process of winnowing down the choices, there will be a few winners, but many, many more losers. The process tends to open up rifts within the community between inner and outer solar system, or astrobiology vs physical science. With Armada, there will be much more science, and much more varied science to spread around. Because Armada takes advantage of the efficiencies inherent in the production, management, and operation of a set of similar vehicles, the fraction of total mission cost that will be available for science will be higher than that for traditional NASA missions. To keep Armada staffed will create many more PI's, Deputy PI's, Co-I's and graduate students than conventional NASA missions. The net result will be a much more intellectually diverse and experienced community, with greater opportunities for early-career scientists. 
As evidenced by the Discovery and SIMPLEx programs, there are dozens of compelling objectives and destinations that are now within the reach of a medium sized spacecraft in the $200 \mathrm{~kg}$ wet mass range. Imagine an Armada program that provides a standard high-performance exploration spacecraft capable of carrying $>25 \mathrm{~kg}$ of payload, powered by a gridded ion thruster, with $8 \mathrm{~kW}$ of power at $1 \mathrm{AU}$, with up to $100 \mathrm{~kg}$ of zenon. Such a spacecraft could fly from low Earth orbit to low Mars Orbit and back again. This requires a long lived, high reliability, single string spacecraft that can be deployed from a 15 in ESPA ring.

An Armada spacecraft concept developed by Deep Space Systems is shown below.
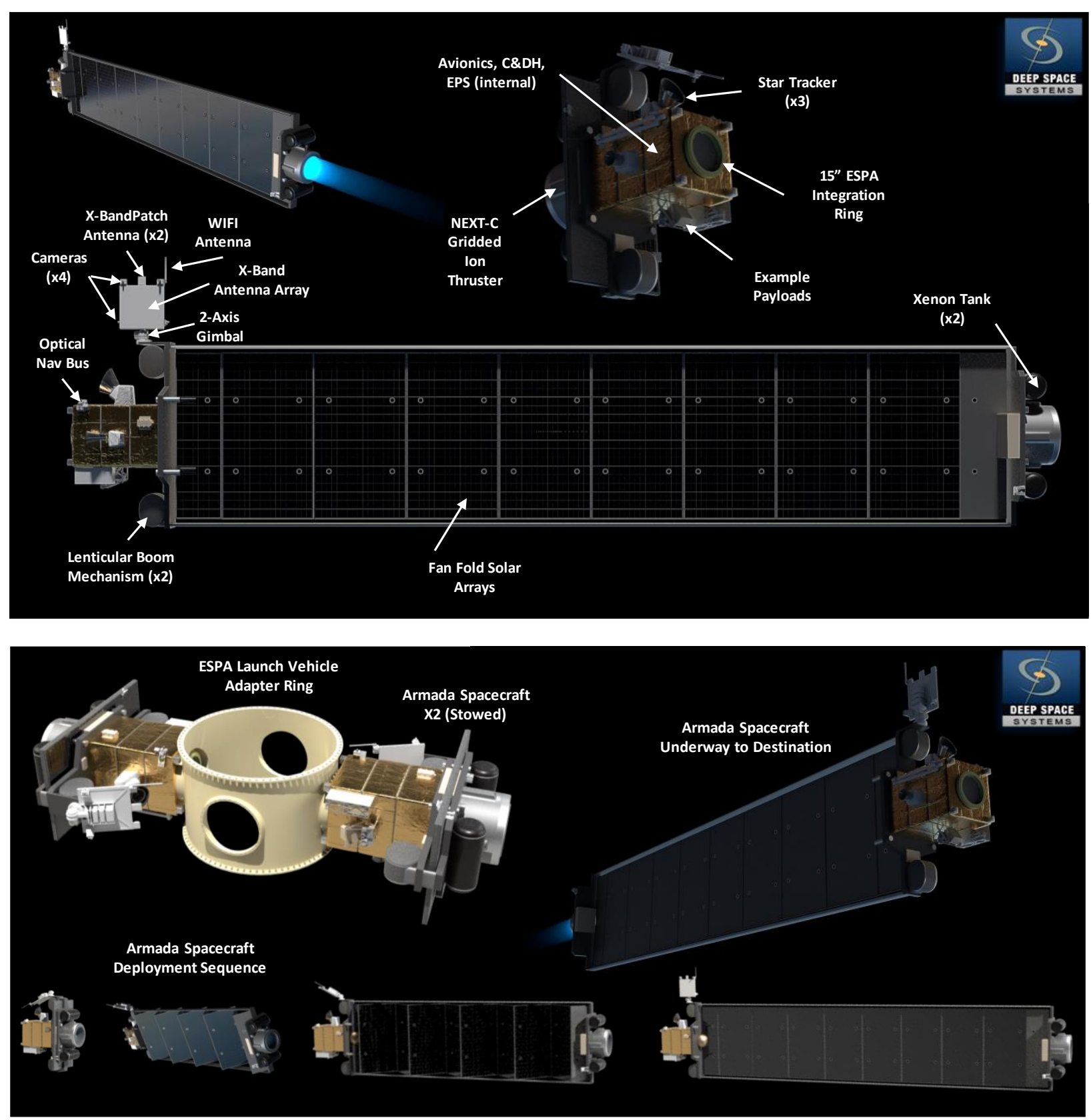

This concept spacecraft design includes a rigid overall spacecraft structure created by the deployment of twin lenticular booms. Solar pressure is balanced by and aft body propulsion module and the forebody 
providing an open platform for science instruments that includes an articulating high gain antenna with integrated cameras. Attitude control is provided by thrust vector control of the main engine, skew reaction wheels fore and aft, and thrusters fore and aft. This concept design is the basis for the mission illustrations that we believe are only scratching the surface of the Armada proposals that will be received as a result of an Announcement of Opportunity for Armada Science Investigations.

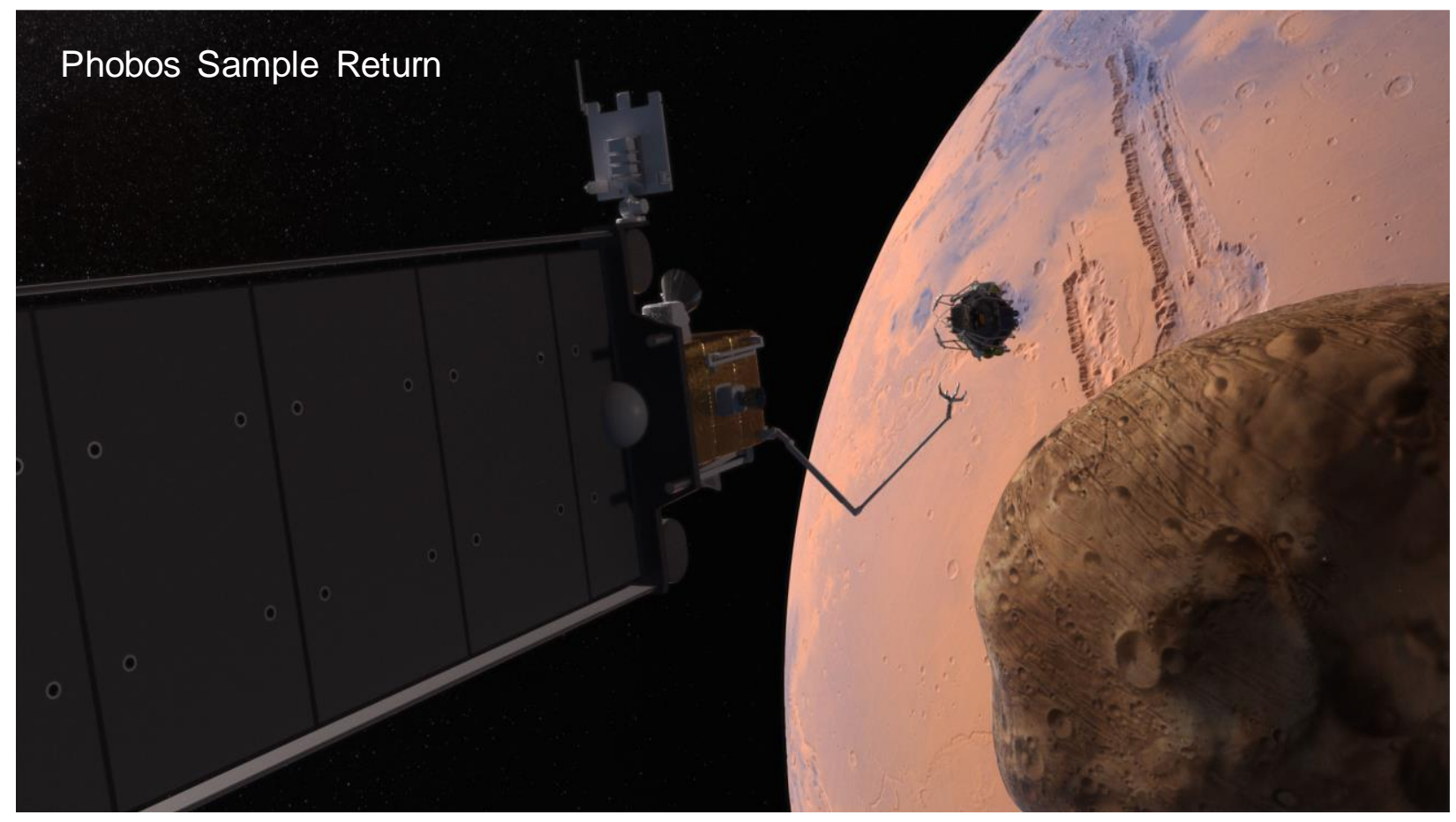

An Armada Flagship Program is not meant to replace or diminish SIMPLEx. Teams competing for SIMPLEx are struggling to produce CubeSat-sized spacecraft with meaningful science payloads, and are pitted against the inherent low reliability and limited capabilities of the CubeSat supply chain as well as the formidable complexity of integrating and operating a one of a kind spacecraft in deep space. The complexity of these missions is largely independent of the size of the spacecraft, and cost estimation here is as much an expression of risk tolerance as anything else. SIMPLEx is an extremely valuable program for pushing the limits of what small missions can accomplish and for bringing innovation and new players into the exploration community. There should be more SIMPLEx funding not less.

Likewise, this proposed Flagship program is not meant to undermine the Discovery or New Frontiers Programs, for the similar reasons, and for the fact that Armada can further energize these already incredibly successful and productive programs, with capabilities and reconnaissance data, allowing New Frontiers class objectives to be accomplished within a Discovery budget.

NASA's most ambitious science missions, the Flagship missions, have produced wonderful science returns, but have consistently experienced budget overruns that have impacted other missions and diminished confidence in NASA's ability to execute programs within their budgets. Many of us believe there are perverse and structural motivations and incentives that make budget overruns in Flagship programs almost inevitable. This is not due to poor "cost modeling". Because these are "Flagship" missions, they MUST succeed. To the executing organizations, mission success is simply more important than cost control. Flagship missions are ambitious, and therefore have more inherent risk than most. 
There is no amount of spending that will reduce risk to zero, but these programs tend towards the absolute limit of spending. In practice, actual spending is governed by the current threshold of political will and institutional pain that NASA, the Congress and other stake holders are willing to tolerate. This simple dynamic governs behavior and will not be broken by the most sophisticated cost modeling tool. There have been no significant consequences for these cost overruns, thus no real incentive for change. But this may change for the next Flagship program. Program cancellation must be seen as a real threat in order to have any real effect on these programs.

There is a way to avoid this dilemma. Instead of a picking a single Flagship spacecraft, we should send out an armada of small spacecraft, where the size of the fleet is defined by a Flagship-sized budget. Cost control is intrinsic to the use of a high TRL small spacecraft and existing infrastructure. The core spacecraft, launch, telecommunications, Earth-relative navigation, and housekeeping flight operations are provided as a service by the Armada program. Dozens of government fleet satellite and commercial constellation operations all over the world today are a testament to just how cost effective it is to design, build, test and operate multiple systems in parallel.

For any given element of the fleet, that is any Armada Project, the PI for that science mission is given hard budget constraints with program enforced margin, descope and cancellation criteria.

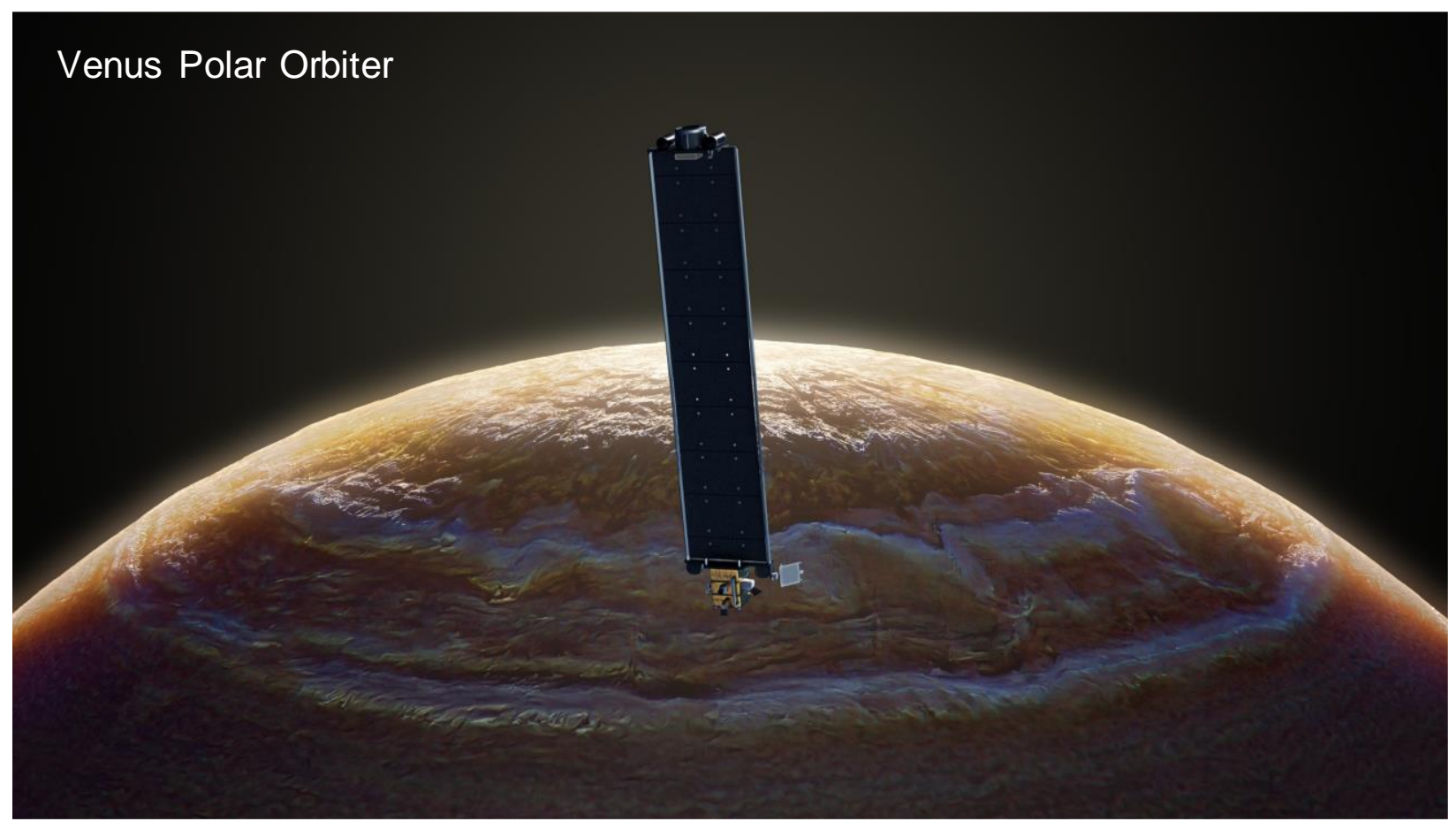

Instead of planning for a Flagship mission that repeats the pattern of cost overrun, we should plan for a \$3 Program cost cap, build and thoroughly test a high reliability common spacecraft and allow science teams to simply add payloads and mission unique modifications. We should provide a common deep space communications, navigation and operations infrastructure for Armada that allows us to fly throughout the solar system to limits of the spacecraft and the ingenuity of the teams.

The non-recurring cost for the spacecraft can be capped at $\$ 150 \mathrm{M}$, the NRE for the ground system at $\$ 50 \mathrm{M}$, the bulk buy of twenty ESPA ride share slots of $200 \mathrm{~kg}$ each should cost less than $\$ 30 \mathrm{M}$, recurring 
cost for 20 standard high-performance, high-reliability, single-string spacecraft as a bulk buy should be no more than $\$ 30 \mathrm{M}$ per spacecraft. This turns the standard mathematics of a Flagship program on its head, where launch is a trivial part of the cost, the DDT\&E for spacecraft and operations through launch are less than $1 / 3$ the budget and over $2 / 3$ of the budget and spending is on the science teams, science instruments, and mission operations. The fleet health and maintenance and uplink/downlink support for the armada, program level management and outreach should cost about \$20M per year.

Each team is responsible for mission unique planning and analysis, instruments, team members, mission support and data distribution and analysis within their $\sim \$ 50 \mathrm{M}$ budgets.

Some missions will fail to get to the launch pad, some may fail in flight, but overall, the program level technical risk, schedule risk and cost risk are very low and are bounded. Due to previous and ongoing NASA investments, the spacecraft technology and ground infrastructure are very high TRL. There is very low risk that science team members will grow old and die during a lengthy development program followed by schedule delays and associated cost growth - and the ever-growing danger of complete program cancellation by disgruntled politicians.

Initial launches should be able to start within 3 years of program start. Each of the 20 missions can launch when ready to LEO, SSO or GTO as a starting point for spiraling out to their respective destinations. This $\mathrm{N}+1$ approach provides great resiliency and graceful degradation at a program level, and if, ultimately, only 16 of 20 missions succeed in achieving their science objectives, the program will still be tremendously successful.

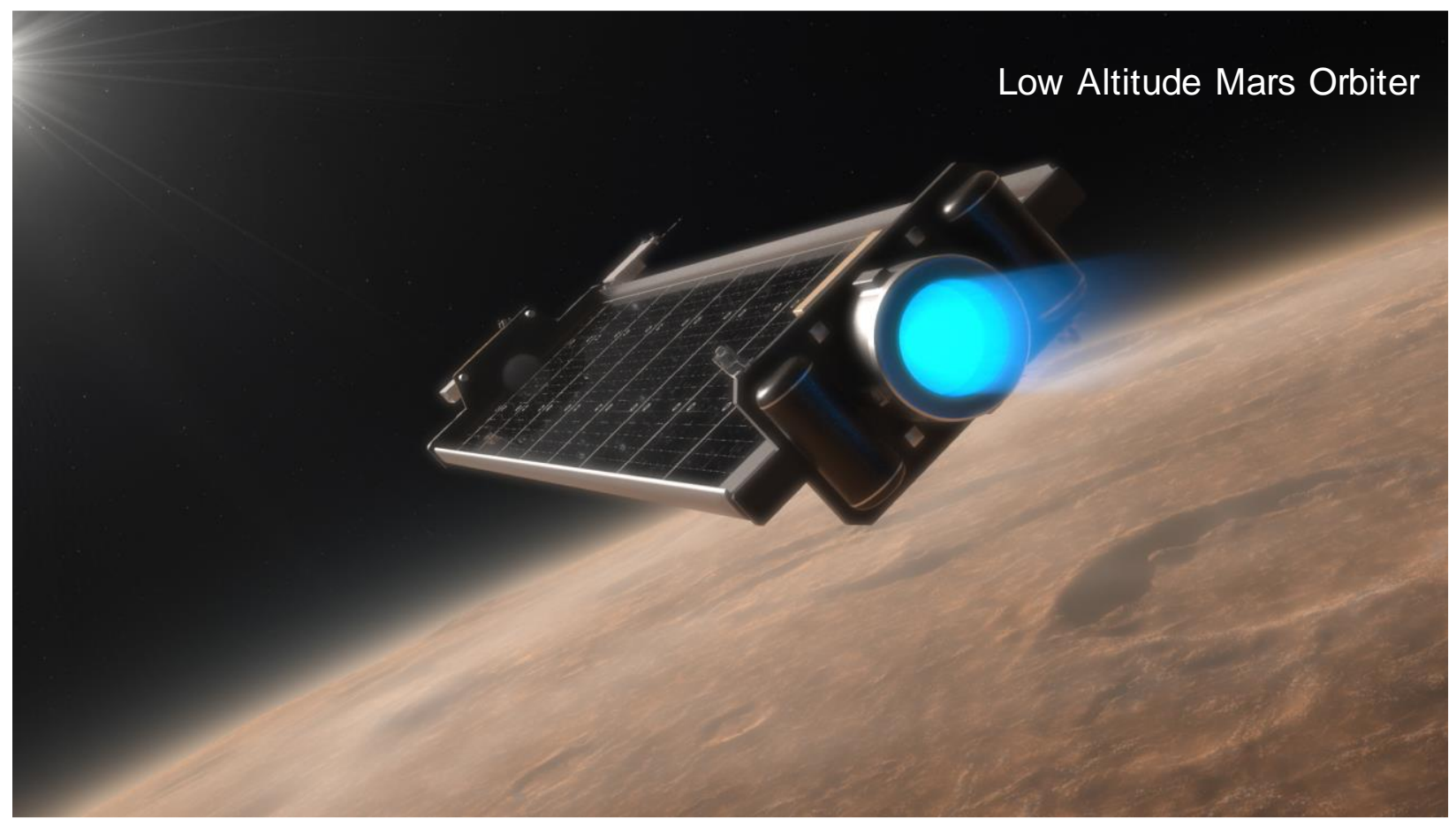

Think of the level of participation and inclusion built into this approach, and think of the fundamental discoveries that occur every time we look closely at the unexplored. What better way to involve and energize scientists, young and old, who yearn for a chance to participate and make discoveries?

We urge you to join us in advocating this fresh approach to a NASA Planetary Science Flagship Program. 